\title{
RFID: Recognizing Failures In Dressing activity
}

\section{Evaluation of Two Methods for Inferring the Order of Dressing}

\author{
Kyriaki Kalimeri ${ }^{\ddagger}$, Aleksandar Matic ${ }^{\S}$, Alessandro Cappelletti ${ }^{\ddagger}$ \\ ${ }^{\ddagger}$ Fondazione Bruno Kessler, Povo, Trento, Italy \\ ${ }^{8}$ CREATE-NET, Povo, Trento, Italy \\ \{kalimeri, cappelle\}@fbk.eu \{aleksandar.matic\}@ create-net.org
}

\begin{abstract}
In elderly care the manual assessment of Activities of Daily Living (ADLs) is a significant problem. Focusing on the dressing we aim not only to recognize the activity performed but also to evaluate its quality, using solely RFID technology. Our approach leverages sparse and noisy RFID readings by applying the two probabilistic methods, Bayesian Networks and Layered HMM. The goal is to follow the garments that patients are manipulating and to detect possible failures such as forgetting to put on something or putting clothes in wrong order. The experimental results showed that both methods can achieve high accuracy.
\end{abstract} RFID;

Keywords: Monitoring ADL; Assessing Dressing Activity;

\section{INTRODUCTION}

ADL (Activities of Daily Living) is a term that encompasses the common tasks that people do in everyday life such as eating, bathing, dressing, toileting and other similar activities. Impaired ability to perform ADLs can be considered as a synonym for loosing independence of autonomous living and it usually leads to a need of having a constant home care or to moving to specialized healthcare institutions (e.g. geriatric residences). Numerous are the causes that affect the performance of ADLs such as strokes, dementias, paralyses, physical injuries and so on. One of the most common factors is certainly ageing which increases the chances for cognitive decline; the prevalence of dementia doubles every five year in patients over the age of 65 [18]. This, in conjunction with the fact that the elderly population is growing, explains the predictions that a number of dementia patients will increase to 42 million by 2020 and to 81 million worldwide by 2040 [17].

In order to mitigate a home care of individuals with impaired ability to perform ADLs and to relieve some stress from healthcare institutions that are faced with increased care provisioning demand, many research groups work on technological solutions for monitoring ADLs [23, 2, 3] to provide tools for assisting and evaluating the quality of performed activities.

In such monitoring systems Radio Frequency Identification (RFID) very often finds its usage, mainly due to the small size of tags and low cost. The technology is convenient for subjects' identification and presence detection in certain areas in the environment covered with RFID antennas. However, in order to categorize performed activities RFID technology is mostly

PERVASIVEHEALTH 2010, March 22-25, Munchen, Germany

Copyright $\odot$ 2010 ICST 978-963-9799-89-9

DOI 10.4108/ICST.PERVASIVEHEALTH2010.8896 used to detect which objects are being manipulated, e.g. in [2, $13,15]$.

This paper presents an approach to evaluate the dressing, one of the basic everyday activities using only RFID technology. There are the systems in the literature that are able to recognize when subjects are dressing/undressing themselves $[2,24]$ but to the best of our knowledge there is no any system capable of recognizing if the task is performed correctly or not. Our objective is to recognize if clothes are put on and in which order. Putting on clothes in incorrect order (e.g. shirt over pullover) is identified as one of the most common dressing failures in dementia patients [20]. Our approach assumes all clothes to be tagged and the recognition of dressing steps is based on clothes presence detection in wardrobe, hanger and in dressing cabin in front of a mirror. The project was developed in accordance with a scenario that was made from observations in an actual rehabilitation center.

Due to the presence of water or metallic objects that absorb radio waves, RFID can provide sparse and noisy readings. In addition, depending on a setup there are the potential issues with antennas reading ranges that could overlap and create noise or not to be enough to detect passive tags on a certain distances. In order to overcome these problems, we analyze two different probabilistic methods, Layered Hidden Markov Model (LHMM) and Bayesian Networks.

\section{RELATED WORK}

During the last years, a lot of research has been carried out towards the systems for AAL (Ambient Assisted Living) and ADL (Activities of Daily Living) recognition. Human action recognition is the key requirement towards this direction and can be divided in four categories: initialization, tracking, pose estimation and recognition. This paper presents work that belongs to the last category; we are interested in recognizing human behaviors that have a particular function: to accomplish a specific task in an ADL.

In similar line with our work is the project Memory Mirror [1], an RFID based project that is focused on people with earlystage cognitive decline. The goal is to reflect the use of specified objects during a certain period of time, while also warns of possibly lost items that have to be returned. Another important work on ADL recognition is MedTracker, presented in [3]. MedTracker is also an RFID system that helps the cognitive impaired patients to take their medication correctly (e.g. usual mistake is taking the same medication twice or 
never) and on time. This system has the ability of individual pill detection. It features an innovative approach that eliminates human intervention in handling the medicine between the pharmacy and the patient pill dispenser. Caregiver's Assistant is a system that monitors the elderly in their own homes [6, 7], requiring from the elderly to keep a high frequency RFID reader on themselves, while several objects are tagged (mainly the ones that subjects have daily interaction with). By comparing the real time recordings and the data already stored in the base it is possible to track the differences in activity patterns.

However, there are two main issues that are emerging in monitoring systems that rely on RFID technology. Firstly, it is highly important to have a reasonable and flexible activity representation. In this direction, Philipose et. al [2] proposed the Proactive Activity Toolkit (PROACT), a toolkit that represents activities as a probabilistic sequence of used objects and then it automatically creates the probabilistic models of activities. Another similar approach is the research on MIT's "House_n" project [4]. This project places a single type of object-based adhesive sensor in structurally unmodified homes. Sensor readings are later analyzed for various applicationskitchen design, context sampling, and potentially ADL monitoring using RFID technology. Secondly, the other significant issue arises due to the RFID's sparse and noisy readings. Various works have been trying to overcome this by introducing probabilistic approaches, mostly using dynamic Bayesian network modeling and HMMs. Peters et al [5] have proposed an agent that applies learning techniques to recognize behaviors of persons with dementia using multiple cues in an HMM-based approach. It is a learning technique for visual event recognition in a system that assists people with dementia during the hand washing activity. They have also proved HMM-based approaches to be superior to a simple k-NN recognition scheme. [12], [13], [15] demonstrated that in comparison to other classifiers Naive Bayes and HMM classifiers are more suited for recognizing activities such as sitting, standing and walking, when low-level sensor data is used.

In comparison to the aforementioned projects our system uses RFID technology in order to monitor the dressing activity, recognize its steps and provide the evaluation of the quality of performed activities. The aim is to follow which garments are put on and to check if the priority is respected. Therefore, it presents the support for creating the assistive system in the future that could help dementia patients by indicating mistakes and to suggest the appropriate action, without a help of a caregiver.

In addition, the paper provides a comparative study between two different implementations of the dressing activity recognition, namely Layered HMMs and Bayesian Networks.

\section{HMM METHOD}

\section{A. Dressing Scenario}

The scenario was designed aiming to reflect a real everyday dressing task. The subjects were asked to perform the task of dressing following the correct order and then with a dressing priority mistake (for instance, wearing a pullover under the shirt). We considered a set of three pieces of clothes: t-shirt, pullover and jacket (marked only with one tag) while the three positions were monitored: drawer, hanger and mirror. During each experiment subjects' actions were annotated in order to have a reliable ground truth to evaluate the effectiveness of the probabilistic model.

\section{B. Activity Recognition and System Architecture}

The system developed is based on logical reasoning with information about the activity from an observed subject and a background knowledge base. In our work, the dressing activity is composed of a set of actions and the aim is to detect priority abnormalities. The major components of the system are described bellow:

\section{a) RFID Sensor Network}

In order to overcome the interference between close-by antennas, we chose the distance to be greater than their reading range, positioned in perpendicular orientation in order to make the coverage fraction maximised [21]. Two antennas were placed close to the mirror in perpendicular positions, one was placed on the hanger and one in the wardrobe.

\section{b) AI Agent}

The agent should be aware of intended activities and it reasons on the RFID tag location in a real time. Each garment is treated separately in parallel thread architecture. Our basic assumption is that location sequences are evidence of actions, and then the sequence of actions are composing an activity.

\section{c) Pattern Recognition and Classification}

A two Layered HMM model [22] has been implemented, aiming to deal with two different challenges; noise reduction and semantic analysis. The lower layer acts as a filter to noise and is composed by three identical HMM-1's, with hidden states that represent the antenna readers' location and observation states that represent the location of the reading plus a "missing item" state. The most probable location of each tag is estimated in a time window of $1 \mathrm{msec}$, using the forwardbackward algorithm while the maximum likelihoods are being fed to the next layer as a-priori probability. This layer interprets actions into activities and it is composed of the three observation states that are directly fed from the previous level and two hidden states that represent the final interpretation of the action; that is, a "garment considered dressed" or "garment considered undressed". The time granularity for the parameter estimation is different and set to $1 \mathrm{sec}$.

In Figure 1 the layer on the left side is intended to eliminate sparse and noisy readings assigning miss-readings to the most probable physical location. All the probabilities are passed to the next layer, on the right, that interprets the action performed according to the sensor readings. Each block corresponds to a level and each block consists of $\mathrm{K}$ HMMs running in parallel. Each HMM has T observation symbols. At any given level $\mathrm{L}$ in the LHMM a sequence of TL observation symbols $O_{L}=\left\{o_{1}, O_{2}, \ldots, O_{T L}\right\}$ is used to classify the input into one of $\mathrm{KL}$ classes. Then the most probable HMM is selected as an observation symbol. 


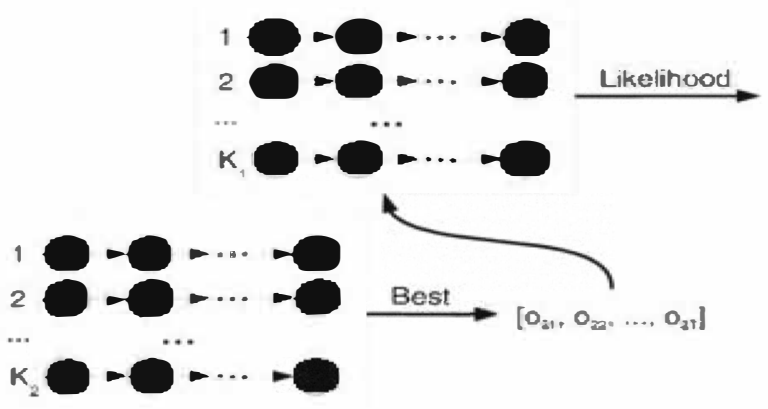

Figure 1 Graphical Representation of our Layered HMM Architecture.

\section{BAYESIAN NETWORK METHOD}

\section{A. Experimental Setup and Dressing Scenario}

In our second method RFID antennas were positioned only in the dressing cabin, two antennas attached on the side walls covering shoulder areas and one on the back wall covering subject's back when he/she is facing a mirror that is supposed to be on the door of dressing cabin (like in clothing stores usually). Adjacent antennas (side antennas and the back one) were on the different heights in order to avoid interference; the side antennas were on $140 \mathrm{~cm}$ and the back antenna on $90 \mathrm{~cm}$ from the ground. Correspondently, clothes were tagged with three tags - two on the shoulders and one on the back. Participants were offered to choose the garments out of t-shirt, pullover, light jacket and winter jacket and to perform dressing both in correct and incorrect order. They were not required to follow any other instruction which had a goal to increase their freedom to perform the task in a natural and random way. They could use a wardrobe, hanger, bed and chair to lie down the clothes during the task.

\section{B. Bayesian Network Model}

We associated each of antennas with nodes in our Bayesian Network Model that are parental nodes to the "FinalState" one which reflects the temporal result of a dressing task, taking either "Put on" or "Not put on" states (Figure 2). "Left" and "Right" nodes can take "Left tag detected", "Right tag detected" and "Nothing detected" states, thus ignoring the back tag. Similarly, "Back" node takes "Back tag detected" and "Back tag not detected" states, ignoring shoulder tags. In that manner, a part of the problems with noisy readings usually caused by holding garment in the dressing cabin is overcome effectively. Once the back antenna reads the back tag and the side antennas read corresponding shoulders tags, an event is categorized as "Put on", concerning the current manipulated garment. Otherwise the "FinalState" node holds "Not put on" state. The probabilities assigned to these states depend on the states of parental nodes and they are adjusted from training evidence. It should be noted that the detection of the back tag and only one of the shoulder tags on the corresponding antenna is sufficient to presume that the garment is put on. Tagging both shoulders just increases the probability for correct dressing steps recognition in case of misreading on one of side antennas. To test this presumption, we tagged two garments with three tags (t-shirt and pullover) while two other ones (light jacket and winter jacket) with two tags (left shoulder and back).

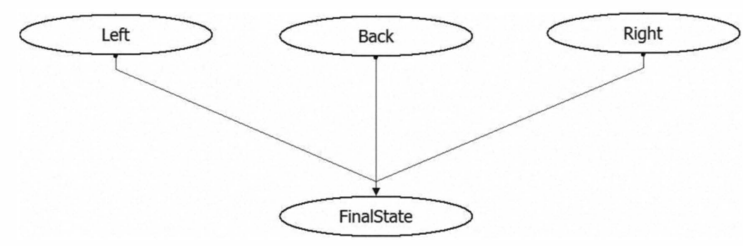

Figure 2 Bayesian Network Model

\section{EXPERIMENTAL RESULTS}

In the experiments, twelve subjects performed in total, twenty one trials of dressing activity for testing HMM, while for Bayesian Network method another twelve persons performed twenty three trials. Table 1 shows the accuracy of recognition for each of the garments and for the detection of correct and incorrect order of dressing.

\section{1) $H M M$}

Leave-one-out cross-validation is performed to evaluate the accuracy of the classification and consequently the stability of the entire agent. Twenty in twenty one trials are used as training data, and the remaining trial is used as verification data. The different error rate between the garments is a consequence of the dressing priorities (e.g. the tag from jacket or pullover that is put over a t-shirt can hide the tag from t-shirt so the probability of misreading is higher).

\section{2) Bayesian Network}

Bayesian Network method also overcame the problems of noisy and sparse RFID readings that were even more common than in first method due to the fact that the garments were tagged with two or three tags and the dressing cabin was covered with three antennas. As expected higher recognition rate is achieved in the experiments with the garments that were tagged three times (t-shirt and pullover) instead of two (light and winter jacket) - Table 1 .

False negatives were the consequence of misreading tags on appropriate antennas: only when one of the shoulder tags and

\begin{tabular}{|c|c|c|c|c|c|c|c|c|}
\hline \multirow{2}{*}{ Task } & \multicolumn{2}{|c|}{ False Positive } & \multicolumn{2}{c|}{ False Negative } & Total Actions Recorded & \multicolumn{2}{c|}{ Accuracy (\%) } \\
\cline { 2 - 9 } & BN & HMM & BN & HMM & BN & HMM & HMM & BN \\
\hline Shirt & 0 & 0 & 2 & 2 & 25 & 24 & $83.3 \%$ & $92.00 \%$ \\
\hline Pullover & 0 & 0 & 0 & 0 & 19 & 16 & $100.00 \%$ & $100.00 \%$ \\
\hline Light Jacket & 0 & 0 & 4 & 2 & 17 & 40 & $95.00 \%$ & $76.47 \%$ \\
\hline Winter Jacket & 0 & 0 & 2 & - & 16 & - & - & $87.50 \%$ \\
\hline Correct Order & 0 & 0 & 2 & 1 & 13 & 11 & $91.00 \%$ & $84.62 \%$ \\
\hline Wrong Order & 0 & 0 & 2 & 1 & 10 & 10 & $90.00 \%$ & $80.00 \%$ \\
\hline
\end{tabular}


the back tag were read at the same moment we considered that a single garment is correctly put on; misreading one of these tags in the case when the garment is put on resulted in false negative. It is unlikely to detect all the tags with corresponding antennas in the case when a garment is not put on; that is why we did not encounter any false positive.

\section{3) Discussion}

According to the Table 1 the LHMM approach gives higher accuracy results. In addition, it does not require multiple tagging of clothes as in the case of the approach that uses Bayesian Network method. However, tagging clothes three times and positioning three antennas at corresponding places in a dressing cabin gives more relieble detection if a garment is put on. For instance, in some cases when a subject just holds a garment in front of mirror it may happen that the HMM method approach reports that the garment is put on, which would not be the case for Bayesian Network method. Depending on specific needs, one may choose one of these approaches by making a trade-off between the accuracy, tagging effort and reliability.

\section{CONCLUSION}

We demonstrated high accuracy of the RFID system aimed to evaluate the dressing activity of dementia patients. We proved that the drawbacks of RFID technology such as noisy or missed readings usually caused by limited reading range, sensitivity to water or interference with metal objects, could be effectively overcome using probabilistic approaches.

A future challenge remains the online learning that is a system that would ideally be capable of adjusting the internal model in real-time as new examples of activities become available. This will allow the algorithm to adapt to changes in the user's routines over time.

\section{ACKNOWLEDGMENTS}

The research was funded by the Autonomous Province of Trento, Call for proposal Major Projects 2006 (Project ACube)

\section{REFERENCES}

[1] C. D. Kidd, R. J. Orr, G. D. Abowd, C. G. Atkeson, I. A. Essa, B. MacIntyre, E. D. Mynatt, T. Starner, and W. Newstetter, "The Aware Home: A Living Laboratory for Ubiquitous Computing Research", In the Proceedings of the Second International Workshop on Cooperative Buildings - CoBuild'99, Position paper, October 1999.

[2] M. Philipose, K. P. Fishkin, M. Perkowitz, D. J. Patterson, D. Fox, H. Kautz, and D. Hahnel, "Inferring Activities from Interactions with Objects", IEEE Pervasive Computing, October 2004.

[3] J. Wu, A. Osuntogun, T. Choudhury, M. Philipose, and J. M. Rehg, "A scalable approach to activity recognition based on object use," In Computer Vision, 2007.

[4] MIT House_n, http://architecture.mit.edu/house_n/, Accessed: December 2009.

[5] C. Peters, S. Wachsmuth, and J. Hoey, "Learning to recognize behaviors of persons with dementia using multiple cues in an HMM-based approach", In Proceedings of the 2nd international Conference on
Pervsive Technologies Related To Assistive Environments, PETRA'09, ACM, New York, NY, 1-8. http://doi.acm.org/10.1145/1579114.1579179, 2009.

[6] Wired-MedTech, "RFID Keeps Track of Seniors" http://www.wired.com/medtech/health/news/2004/03/62723 , Accessed: December 2009.

[7] Intel Labs Seattle, http://seattleweb.intel-research.net/, Accessed: December 2009.

[8] RFID Journal, "New System Reports Patient Falls", http://www.rfidjournal.com/article/articleview/2462/1/1/, Accessed December 2009.

[9] M. Philipose, K. Fishkin, M. Perkowitz, D. Patterson, D. Fox, H. Kautz, and D. Hahnel, "Inferring activities from interactions with objects", IEEE Pervasive Computing, 3(4):50- 57, 2004.

[10] L. Bao and S. Intille, "Activity recognition from user-annotated acceleration data", In Pervasive, volume 3001 of Lecture Notes in Computer Science, pages 1-17, 2004.

[11] N. Ravi, N. Dandekar, P. Mysore, and M. L. Littman. "Activity recognition from accelerometer data", In AAAI, pages 1541-1546, 2005.

[12] J. Ward, P. Lukowicz, and G. Troster, "Gesture Spotting Using Wrist Worn Microphone and 3-Axis Accelerometer," in sOc-EUSAI, 2005.

[13] N. Ravi, N. Dandekar, P. Mysore, and M. Littman, "Activity Recognition from Accelerometer Data", In Proc. IAAI, 2005.

[14] T. B. Moeslund, A. Hilton, and V. Kruger, "A survey of advances in vision-based human motion capture and analysis", Computer Vision and Image Understanding, 104(2-3):90-126, November-December 2006.

[15] M. Stikic, T. Huynh, K. V. Laerhoven, B. Schiele, "ADL recognition based on the combination of RFID and accelerometer sensing", Pervasive Computing Technologies for Healthcare, 2008.

[16] M. D. Shulman, "Maximizing communication with the Alzheimer's patient", Nursing Homes. FindArticles.com, 09 Dec, 2009.

[17] C. P. Ferri, M. Prince, C. Brayne, H. Brodaty, L. Fratiglioni, M. Ganguli, K. Hall, K. Hasegawa, H. Hendrie, Y. Huang, A. Jarm, C. Mathers, P. R. Menezes, E. Rimmer, M. Scazufca, "Global Prevalence of Dementia: Delphi Consensus Study", Lancet, 366, 2112-17, 2005.

[18] J. Lindsay, E. Sykes, I. McDowell, R. Berreault, and D. Laurin, "More than the epidemiology of Alzheimer's disease: Contributions of the Canadian Study of Health and Aging", Can J. Psychiatry, vol. 49, no. 2, pp. 83-91, 2004.

[19] X. H. B. Le, M. D. Mascolo, A. Gouin, and N. Noury, "Health Smart Home - Towards an assistant tool for automatic assessment of the dependence of elders", Proceedings of the 29th Annual International Conference of the IEEE EMBS Cite Internationale, Lyon, France, August 23-26, 2007.

[20] A. Lobay, D. A. Forsyth, "Recovering Shape and Irradiance Maps from rich dense texton fields", Proceedings of the IEEE Computer Society Conference on Computer Vision and Pattern Recognition, 2004.

[21] Q. Yang, "Activity Recognition: Linking Low-Level Sensors to HighLevel Intelligence", International Joint Conference on Artificial Intelligence [Online], 24 Jun, 2009

[22] N. Oliver, A. Garg, and E. Horvitz, "Layered representations for learning and inferring office activity from multiple sensory channels". Comput. Vis. Image Underst. 96, 2, 163-180. DOI= http://dx.doi.org/10.1016/j.cviu.2004.02.004, 2004.

[23] A. Mihailidis, B. Carmichael, and J. Boger, "The Use of Computer Vision in an Intelligent Environment to Support Aging-in-Place", Safety, and Independence in the Home, IEEE Transactions on Information Technology in Biomedicine, Vol. 8, No. 3, September 2004.

[24] A. F. Dalton, F. Morgan, and G. O. Laighin, "A preliminary study of using wireless kinematic sensors to identify basic Activities of Daily Living", $30^{\text {th }}$ Annual International IEEE EMBS Conference, Vancouver, British Columbia, Canada, 2008 